\title{
Entre la vida y la muerte: el dolor. Un acercamiento foucaultiano ${ }^{1}$
}

\author{
Between life and death: pain. \\ A Foucauldian approach
}

Dulce María Cabrera Hernández ${ }^{2}$

Benemérita Universidad Autónoma de Puebla, México

Recepción: 15 de julio del 2021

Evaluación: 8 de septiembre del 2021

Aceptación: 23 de septiembre del 2021

1 Este artículo corresponde al proyecto PAPIIT-UNAM: IN305521 "Ética y Biopolítica. Prácticas socioeducativas universitarias sobre tecnología y salud".

2 Doctora en Pedagogía por la Universidad Nacional Autónoma de Puebla.

Correo electrónico: dulcemariacabrera@gmail.com 


\title{
Resumen
}

Este trabajo se propone, desde una perspectiva foucaultiana, reflexionar acerca de la incorporación del dolor en una ética de la resistencia. En el artículo se revisan algunos planteamientos relacionados con el nacimiento de la clínica y las tres conferencias ofrecidas por Foucault durante 1974 en Río de Janeiro, para analizar el abordaje de la vida, la enfermedad y la muerte, y el gobierno de los cuerpos. El argumento central es que el discurso de la medicina poco o nada puede hacer con el dolor, porque éste se constituye en un espacio intermezzo entre la vida y la muerte. Aunque el dolor amenaza el despliegue de la positividad de la vida y de la salud plena, no conduce a su aniquilación. En este sentido, es posible pensar en una ética capaz de oponer resistencia a la industria del fármaco o al mercado de la salud, y apostar por una cierta experiencia que ayude a reconocer el dolor como un proceso colectivo.

Palabras claves: ética, filosofía, salud, sistema social.

\begin{abstract}
From a Foucauldian perspective, this paper is addressed to trigger a reflection about the incorporation of pain into an ethic of resistance. In this paper, some ideas related to the birth of the clinic and other three conferences offered by Foucault in 1974, in Rio de Janeiro Brazil, are reviewed to analyze the approach of life, sickness, and death, and the bodies' governance. The main argument affirms Medicine's discourse can do very little or nothing against pain, because it is an intermezzo space between life and death. Although pain threatens the deployment of positivity of life and health, it cannot conduct to their extermination. In such vane, it is possible to think about an Ethic able to resist against pharma industry or health business and to bet for an exceptional experience that helps us to recognize pain as a collective process.
\end{abstract}

Keywords: ethics, philosophy, health, social system. 


\section{La vida, la enfermedad y la muerte}

El poeta es un fingidor / Finge tan completamente que hasta finge que es dolor, el dolor que de veras siente / Y quienes leen lo que escribe, sienten, en el dolor leído, no los dos que el poeta vive sino aquél que no han tenido (Pessoa, Antología poética, p. 132).

Este escrito inicia reconociendo que la vida, la enfermedad y la muerte han estado históricamente en el análisis de los sistemas de verdad, saber, poder y gobierno. Por ejemplo, en La historia de la locura en la época clásica, publicada en 1961, Foucault (2015) afirma que se consideraba al loco como un hijo del mar, el bobo y el necio fueron parlanchines y vagabundos durante el siglo XVII. Hacia el XVIII surgió la figura del enfermo peligroso y la desgarbada locura, dueña de las calles, fue confinada y desplazada por la razón: "En todo caso, la historia de la locura no es una trayectoria de la razón hacia la verdad, sino que es una progresiva dominación de la locura para integrarla en el orden de la razón (...)" (Machado, 1999, p. 21).

En El nacimiento de la clínica. Una arqueología de la mirada médica, Foucault (2006) expone que la vida se presenta amenazada por la enfermedad y la muerte. Lo que interesa al autor en esa obra es el discurso de la medicina en su relación con la clínica. Valiéndose del médico y de la idea de cuerpo enfermo, se muestra la emergencia de la medicalización: "La clínica figura, por lo tanto, como una estructura esencial para la coherencia científica, pero también para la utilidad social y para la pureza política de la nueva organización médica (...)" (Foucault, 2006, p. 107). En la obra mencionada se encuentra un capítulo dedicado a la creación y funcionamiento de los hospitales. Se afirma en ella que estas instituciones jugaron un papel central en la organización del conocimiento médico, en la transmisión de ese saber y en la habilitación profesional de ciertos sujetos para intervenir, hablar y ver claramente los rasgos de la enfermedad y de la muerte. Con esos elementos se estructuró el discurso de la medicina y se registró una racionalidad, un lenguaje y una gramática:

Esta nueva estructura está señalada, pero por supuesto no agostada, por el cambio ínfimo y decisivo que ha sustituido la pregunta: "¿Qué tiene usted?", con la cual se iniciaba en el siglo XVIII el diálogo del médico y del enfermo con su gramática y su estilo propios, por esta otra en la cual reconocemos el juego de la clínica y el principio de todo su discurso: “¿Dónde le duele a usted?" (p. 14). 
La creación de códigos, lenguajes, credenciales y procedimientos para intervenir en los cuerpos enfermos alimentó una pedagogía de la medicina o una pedagogización médica que instituyó un campo de conocimientos especializados en el que participaron médicos y oficiales de la salud. Esta pedagogía se hizo indispensable para acceder a una jerga especializada con la cual nombrar distintas enfermedades, principalmente de los desfavorecidos que, víctimas de la guerra o de la pobreza, eran internados en los hospitales. A cambio de servicios médicos gratuitos, ellos donaron sus cuerpos para que se experimentara con sus padecimientos.

En ese periodo el hospital y la clínica se legitimaron como espacios para trabajar y expoliar el cuerpo. A través de la experimentación se logró identificar, clasificar y organizar el conocimiento médico sin reparar demasiado en la violencia ejercida sobre los cuerpos convertidos en pizarras humanas. Quienes buscaban alivio aceptaban el dolor o la exhibición como moneda de cambio. De esta manera “(...) la mirada clínica tiene esa paradójica propiedad de entender un lenguaje en el momento en que percibe un espectáculo (...)" (p. 155).

En este punto es interesante reiterar la relación y el reforzamiento que existe entre la clínica y la pedagogía. La primera alude al entorno donde la enfermedad se hace visible y tratable dentro del hospital, mientras que la segunda se refiere a mecanismos, dispositivos y tratamientos de las enfermedades. De acuerdo con Foucault, en la observación clínica confluye el ámbito hospitalario y el dominio pedagógico, porque en ella se sintetiza el acto de conocer y se modulan prácticas sociales al transmitir una episteme.

¿Cuál es la importancia que adquiere la observación clínica? Podría decirse que la observación es el mecanismo epistémico que permite asociar el síntoma a la enfermedad y lo que ésta significa al interior del discurso de la medicina:

Es la descripción, o más bien la labor implícita del lenguaje, en la descripción que autoriza la transformación del síntoma en signo, el paso del enfermo a la enfermedad, el acceso de lo individual a lo conceptual. Y allí se anuda, por las virtudes espontáneas de la descripción, el vínculo entre el campo aleatorio de los acontecimientos patológicos y el dominio pedagógico en el cual éstos formulan el orden de su verdad (p. 164). 
Entre la vida y la muerte, el discurso de la medicina asume la función de “(...) hacer hablar lo que todo el mundo ve sin verlo, a los únicos que estén iniciados en la verdadera palabra" (p. 166). Describir es el procedimiento que permite ver y saber. No olvidemos que la vía de acceso a la palabra es el ojo -la observación- y el resto de los sentidos. De esta manera el discurso de la medicina identifica un cuerpo enfermo.

En 1966, en Las palabras y las cosas, Foucault (2005) señaló las condiciones históricas que favorecieron la estructuración de un discurso que permite ver, oír y hablar sobre los cuerpos. Foucault indicó que ese discurso instala una grilla o matriz de intelección desde los cuales se nombra la vida, el lenguaje y la cultura. El tránsito hacia una episteme moderna quedó marcado en $L a$ arqueología del saber, libro de 1969 en el que Foucault (1984) sostuvo: "En la época clásica, se tiene, pues, una formación discursiva y una positividad absolutamente accesible a la descripción (...)” (p. 301).

A partir de estas obras, el estudio sobre la vida, la enfermedad y la muerte se hizo acompañar de una disección sobre las relaciones entre poder-saberverdad (Mancilla, 2021). El cuerpo y la enfermedad ya no solo se explican desde el discurso de la medicina, sino que el punto de inflexión fue la producción de verdad a partir de una estructuración discursiva y de relaciones de poder.

\section{La salud y el gobierno del cuerpo}

Las tres conferencias que Foucault dictó en 1974 en el Instituto de Medicina Social, Centro Biomédico de la Universidad del Estado Río de Janeiro (Brasil), muestran elementos centrales del discurso de la medicina en su relación con el cuerpo, la emergencia de una biopolítica $\mathrm{y}$, finalmente, la disciplina.

En la conferencia intitulada la Crisis de la medicina o la crisis de la antimedicina (Foucault, 2018), dictada en octubre de 1974, el autor señaló que sus estudios sobre el gobierno del cuerpo comprenden un plano individual y otro colectivo, que en ese momento denominó somatocracia. Ésta se remonta a las prácticas médicas estatales en la Europa del siglo XVIII y tiene continuidad en las del siglo XX. En concreto, el filósofo francés se refiere al Plan Beveridge (Ravanal y Aurenque, 2018): 
El Plan Beveridge indica que el Estado se hace cargo de la salud. Se podría afirmar que esta no era una innovación, pues desde el siglo XVIII una de las funciones del Estado, si no fundamental por lo menos importante, era la de organizar la salud física de los ciudadanos. Sin embargo, creo que hasta mediados del siglo XX la función de garantizar la salud de los individuos significaba para el Estado, esencialmente, asegurar la fuerza física nacional, garantizar su capacidad de trabajo y de producción, así como la defensa y ataques militares. Hasta entonces, la medicina estatal consistió en una función orientada principalmente hacia fines nacionalistas cuando no raciales. Con el Plan Beveridge la salud se transforma en objeto de preocupación de los Estados, no básicamente para ellos mismos sino para los individuos, es decir, el derecho del hombre a mantener su cuerpo en buena salud se convierte en objeto de la propia acción del Estado (Foucault, 2018, p. 162).

Una función del Estado, entonces, se dirigió a la implementación de una moral del cuerpo porque era menester inculcar hábitos de higiene y cuidado, tanto individual como familiar. Más tarde, con el Plan Beveridge, comenzó a constituirse el derecho a la salud, la economía, el mercado de la salud, la prevención de las enfermedades y el cálculo de los gastos sanitarios. Todos ellos orbitaron alrededor del cuerpo, sin reparar en la historia de este concepto.

El primer rasgo del modelo médico que Foucault visibiliza en esa conferencia se relaciona con tres asuntos: la organización clínica y hospitalaria que se gestó desde el siglo XVIII, el discurso de la medicina cuya autoridad se erigió a partir de una cierta base científica, y con el riesgo a la vida que provocaba la intervención médica:

En la actualidad, con las técnicas de que dispones, [por ejemplo] la medicina, la posibilidad de modificar el armamento genético de las células no solo afecta al individuo o a su descendencia sino a toda la especie humana (...) Surge pues, una nueva dimensión de posibilidades médicas, a la que denominaré la cuestión de la biohistoria. El médico y el biólogo ya no trabajan a nivel del individuo y de su descendencia, sino que empiezan a hacerlo a nivel de la propia vida y de sus acaecimientos fundamentales. Estamos en la biohistoria y este es un elemento muy importante (p. 167).

El segundo rasgo es la medicación indefinida. En el siglo XX la autoridad del discurso médico es suficiente para establecer una intervención científica, técnica y estatal sobre los cuerpos a través de la examinación, siempre al 
amparo de la salud y contra la enfermedad. Sin embargo, el autor insiste en señalar que desde el siglo XVIII se rompió la relación médico-paciente y que el combate a la enfermedad no se hizo para evitar la muerte, sino que devino en preservación de la vida productiva. A partir de entonces, el objeto de la medicina ya no es la enfermedad, ni la muerte, es la salud: un enfermo es un individuo peligroso para sí mismo y para la vida en su conjunto. Ese riesgo social se combate con las normas sanitarias, con los hospitales y con las prescripciones.

Un tercer rasgo es la configuración, desde el siglo XVIII, de una economía política de la medicina, que se originó para controlar las pérdidas ocasionadas por una epizootia en Francia. Lejos de quedarse como particularidad coyuntural, la medicina moderna consiguió instituir la salud como un objeto de consumo, y en esa dirección se observan procesos de producción y distribución de servicios y medicamentos para garantizar la vida. Tal economización condujo a la transformación de los hospitales, pues paulatinamente dejaron de funcionar como lugares de confinamiento para los locos, y de tortura para los anormales, hasta convertirse en laboratorios que estimulan la continuidad de una vida productiva. Con lo expresado en la primera conferencia, Foucault pasó de la somatopolítica a una incipiente biopolítica (tema que aparece en la segunda conferencia).

Raffin resume las características de la medicina moderna señalando que en ella surge una autoridad médica cuyo alcance regulatorio excede a los hospitales. Ese surgimiento ocasiona los siguientes cambios: se reconfigura el campo de la medicina, se prioriza la vida y se estimula la productividad. Los hospitales se consolidan como aparatos de medicalización indefinida y se introducen "mecanismos de administración médica" (2018, p. 113).

En octubre de 1974 se realizó la segunda conferencia de Río de Janeiro, en la que el filósofo analizó el Nacimiento de la medicina social (Foucault, 1999a). El principio está marcado por un resumen respecto de la conferencia anterior: a) la biohistoria como los efectos o huellas de la medicina en la concepción de lo biológico; b) la medicalización como una red que jerarquiza el cuerpo y el comportamiento, estableciendo mecanismos de regulación social que comprenden al individuo y a la colectividad; y c) la economía de la salud que favoreció la incorporación de mecanismos sanitarios (economías, hospitales, laboratorios farmacológicos) en las sociedades modernas. Enseguida se 
expone el carácter social o colectivo de la medicina, la objetivación del cuerpo como realidad biopolítica y la declaración de que la medicina es una estrategia biopolítica (Ravanal y Aurenque, 2018; Castro, 2016 y 2021).

En esta ponencia Foucault sostuvo que hacia el siglo XIX se abandonó la preocupación por la enfermedad para dirigirla al cuerpo como fuerza productiva. Esto se logró con tres modelos: 1) La medicina del Estado alemán orientada a la salud pública, que puso en operación a la policía médica para vigilar, administrar y organizar a los profesionales; 2) La medicina urbana francesa se desarrolló desde el siglo XV al XVIII para evitar los contagios masivos y las infecciones en los primeros centros urbanos, debido a que en esa época los mecanismos de control no fueron establecidos por el Estado. Los hospitales asumieron su diseño e implementaron cuarentenas, crearon medidas de aislamiento social entre la urbe y el campo, y pautaron la circulación restringida, así como la vigilancia de la población obrera y el confinamiento en los barrios; 3) La medicina inglesa dedicada a la fuerza de trabajo, implantó el control sanitario de las clases trabajadoras y pobres, especialmente para separarlas de los ricos. En consecuencia, la medicina y la economía promovieron nuevas leyes para proteger el derecho a la propiedad y a la vivienda (Foucault, 1999a). En el modelo inglés se ofreció asistencia médica, se hizo un registro de sanidad pública y se estableció un control sanitario de los pobres.

En la tercera conferencia, La incorporación del hospital en la tecnología moderna (Foucault, 1999b), el autor retoma el análisis acerca del hospital para mostrarlo como una institución dedicada a la medicalización, ya que hasta la Edad Media esta institución acogía únicamente a los pobres para salvar su alma, y no su cuerpo. Ese lugar de internamiento no contaba con la tecnología necesaria para crear una cura que aliviara los males físicos, por lo que fue necesario crear los nuevos medicamentos para lograrlo. Si esos establecimientos solo eran espacios de encierro y no de cura, entonces, fue necesario medicalizar el hospital. Al respecto, Foucault señala que fue necesario observar la administración hospitalaria y reajustar sus mecanismos para revertir el desorden y contrarrestar los efectos nocivos que éste causaba.

Después de analizar el funcionamiento de los hospitales ubicados en las aduanas y del hospital militar, Foucault llegó a la conclusión de que la medicalización del hospital fue posible gracias a la disciplina. Esa nueva 
técnica de los siglos XVII y XVIII tenía como objeto la gestión del hombre a partir de una distribución espacial, una sincronización de los cuerpos, una vigilancia constante y un registro de los comportamientos (1999b). La conferencia sobre la tecnología hospitalaria cierra con dos afirmaciones de suma importancia: a) durante el siglo XVIII el hospital logró convertirse en una organización que transmite un saber médico a través de la clínica; y b) "Gracias a la tecnología hospitalaria, el individuo y la población se presentan simultáneamente como objetos del saber y de la intervención médica" (p. 110).

\section{¿Y el dolor?}

En las líneas precedentes se ha recuperado con insistencia el estudio de Foucault en torno al discurso de la medicina en el siglo XVIII. También se ha apuntalado el desplazamiento de la atención por el cuidado del alma hacia el gobierno de los cuerpos. En la tercera conferencia se delinearon algunas pistas sobre la emergencia de la disciplina y aparecieron, de manera temprana, algunas ideas que atañen a las relaciones entre el individuo, la población y la biopolítica. En esta revisión interesa enfatizar algunos puntos:

1. Desplazamiento del gobierno del alma hacia el gobierno de los cuerpos.

2. Medicalización de la sociedad, fuertemente relacionada con la creación y administración de drogas y fármacos, lo que implica la emergencia de enfermedades contemporáneas que no se restringen a los códigos y padecimientos biológicos.

3. Expansión del discurso médico y el diseño de una economía de la salud que implica la producción farmacológica.

Todos ellos amalgamados en una red identificable desde el siglo XVIII y XIX, y que lejos de ser anulada ante la celeridad de las transformaciones sociales contemporáneas, se ha potenciado con el desarrollo del sistema capitalista. La enfermedad ya no se inscribe en el régimen de lo biológico (si es que alguna vez lo fue, pues Foucault, precisamente, señala que la inscripción de la enfermedad se hizo posible a partir de la medicina y de la observación), sino que avanza hacia la construcción de una sociedad patologizada al mismo tiempo que enarbola la vida. En síntesis, en el discurso de la medicina nunca se ha tratado de la relación médico-paciente, enfermedad-cura, salud-muerte; en su lugar se ha medicalizado al individuo y la sociedad, privilegiando la positividad de la vida: 
Desde la medicina, se denomina dolor a la "sensación desagradable causada por una estimulación de carácter nocivo de las terminaciones nerviosas sensoriales. Es un síntoma cardinal de la inflamación y es valorable en el diagnóstico de gran número de trastornos y procesos. Puede ser leve o grave, crónico, agudo, punzante, sordo o vivo, localizado o difuso" (Clarós y D’Ángelo, 1994, p. 412; Pérez-Marc, 2011, p. 35).

La cita previa ofrece aparentemente una definición del dolor. Empero, nos interesa descomponer sus enunciados. La primera parte, que hace referencia a la estimulación nociva de las terminaciones sensoriales, se inscribe en una dimensión anatomofisiológica (2011). La segunda parte, el síntoma, es bastante cercano a lo que Foucault ya indicaba en su obra sobre la clínica, pues al identificar y describir la regularidad de los síntomas se habla de “(...) la constancia de las causas, de la obstinación de un factor cuya presión global y siempre repetida determina una forma privilegiada de afecciones (...)" (Foucault, 2006, p. 44). Ahora bien, queremos apuntar un elemento subjetivo que está presente en esta definición sobre el dolor: su agudeza, levedad o localización. Pero ¿qué es lo que el sujeto hace suyo: el dolor como experiencia o la expresión del discurso médico? Al respecto Mujica sostiene lo siguiente: "La experiencia del dolor fija el tiempo en el presente: durante el dolor no experimentamos más que el instante doloroso, la vivencia del dolor parece suspendida a tal punto que nuestro horizonte temporal no puede sobreponerse a la sensación dolorosa y nuestra memoria no tiene otro contenido que el momento de la lesión (...)" (2020, p. 34).

Esta cita puede inducirnos a pensar que el dolor es de uno en el cuerpo propio con su temporalidad particular, pero este factor depende del carácter normativo de la medicina, ya que únicamente un sujeto medicalizado puede reconocer en qué parte del cuerpo -dónde- siente dolor, la intensidad, su ubicación y gravedad.

¿Es posible registrar el malestar del dolor sin asociarlo a una enfermedad o a la muerte? Sanguineti (2019) afirma que un tipo de dolor es el emotivo o afectivo que no se deriva directamente de una sensación, sino que es más cercano a una tristeza o disposición emocional. Un tercer tipo alude al dolor espiritual ligado a la compasión o conmiseración. Desde otra perspectiva, Pro considera que "(...) el dolor deviene sufrimiento cuando se hace autónomo, cuando manifiesta un debilitamiento de la vida contra el cual soy impotente (...)" (2020, p. 379). 
¿Cómo ha sido posible que en las relaciones entre la vida, la enfermedad y la muerte se haya soslayado la importancia del dolor? Una posible explicación se encuentra en la dinámica de las sociedades contemporáneas, en las que el discurso de la medicina promueve y sostiene un sistema de expoliación de los vivos, aunque éstos sufran o se duelan, es necesario seguir alimentando los llamados a la "superproducción del hacer-vivir" (Valle, 2019, p. 117).

\section{Positividad de la vida}

Vemos ahora que no se abandonó el modelo médico identificado por el filósofo francés, sino que convivimos con sus artilugios, es decir, el ocultamiento del dolor, el valor de la salud como objeto de consumo y la estimulación actual de la positividad de la vida ante la muerte: "La mera positividad de los procesos contemporáneos, al haber soterrado a la presencia como mecanismo de control, permite el libre despliegue de la producción en sus múltiples manifestaciones: producción de saber, producción de capital (...)" (Xolocotzi, 2018, p. 21). Esta producción motiva y sobrevalora el poder de la vida, pero no de cualquier vida; únicamente privilegia aquella que alimenta el engranaje de la ganancia, del capital y de la vanidad. Esa positividad parece colmar todos los espacios de la vida biológica y de la espiritual, reinstalando los mecanismos advertidos por Foucault.

¿Es posible aceptar que la positividad de la vida está siempre amenazada por el dolor como una cierta negatividad que impide su despliegue? Nuestro arriesgado argumento supone que el dolor es la principal negatividad que amenaza la plenitud o positividad de la vida. Mientras la muerte representa la extinción de una forma de vida, el dolor parece ser un elemento que la acompaña, la amenaza y la niega, pero que no conduce necesariamente a su aniquilación (Cabrera, 2021). Es decir, el dolor niega la posibilidad de una vida positiva y llena de salud, pero no se convierte en muerte o destrucción. Buyentik había indicado que esta amenaza no es destructiva, es localizable y no atenta contra la integridad del sujeto (Mujica, 2020). Sin embargo, nos gustaría introducir un matiz: el dolor que amenaza la positividad y plenitud de la vida no es un dolor localizado en alguna parte del cuerpo. Cuando decimos me duele una muela, un dedo o una pierna, somos capaces de identificar las sensaciones o los estímulos desagradables, pero hay un cierto límite en el que experimentamos un dolor que no es solo físico, sino que invade toda la existencia del individuo y de una colectividad. 
En este sentido, la capacidad del dolor corporal de llenar el espacio subjetivo -desde la piel hasta lo más íntimo- sólo es sobrepasado con el dolor espiritual que podríamos llamar intersubjetivo. En el duelo se puede encontrar un ejemplo de este dolor más social o colectivo y quizá cercano al sufrimiento. ¿Por qué el dolor en el duelo tiene efectos tan dislocatorios y en qué medida aparece como negatividad o amenaza para la vida? Lo primero ocurre porque el duelo es la huella o rememoración de una pérdida o ruptura, y lo que nos duele en alguna parte de nuestro ser es saber que aquello perdido no podrá recuperarse y que esa hendidura es imposible de suturar. En este punto coincidiríamos con Pro (2020) cuando nos recuerda que el sufrimiento incapacita e impide que seamos funcionales. En la medida en que la restauración de un estado previo a la pérdida es imposible y debido a la dificultad que implica proyectar un futuro, el dolor aparece como amenaza a la positividad de la vida y trastoca la salud (o lo que ella signifique). En otras palabras, el dolor impide que la vida se realice dentro de las pautas biológicas o sociales.

Frente al dolor, el discurso de la medicina poco o casi nada puede ofrecer: en primer lugar, porque privilegió la vida como capacidad productiva; en segundo lugar, porque fabricó a la muerte como un fatal desenlace para los humanos; y, en tercer lugar, debido a que el mercado de la salud se ha intentado lucrar con el dolor, promoviendo su supresión en la vida moderna, vendiéndolo como un factor indeseable y como el principal enemigo de la felicidad y la salud.

¿Es posible pensar que el discurso de la medicina ha operado de manera estratégica haciéndonos creer que se olvidó del dolor o nos engaña fingiendo que lo oculta? ¿Será esta presencia velada del dolor una estrategia para el sostenimiento de la positividad de la vida y del discurso médico?

Una posible respuesta partiría del reconocimiento de que sí, efectivamente, la maquinaria de la medicina pone estratégicamente al dolor en un segundo plano para dejarlo operar a la sombra en la sociedad capitalista contemporánea. Esto significaría que el dolor es una pieza clave del mercado de la salud y que la vida se prolonga a partir de su sometimiento. En esta circunstancia, el dolor podría aparecer como el motor del mercado y de la sociedad productiva. Empero, en este caso, no habría ruptura entre la vida, la enfermedad y la muerte, pues lejos de amenazar la vida, el dolor se sumaría a las empresas 
productivas del capitalismo. Al ver el crecimiento constante de las ventas de productos analgésicos se podría apuntalar esta controversial postura. Los analgésicos se autoadministran para contrarrestar cualquier dolencia y restaurar la funcionalidad del individuo. De esta manera la medicina y los productos farmacológicos operan para extender la vida productiva y la fuerza de trabajo. Ambos integran una empresa corporativa que nutre a una sociedad paliativa que consume medicamentos para distraerse del dolor y del sufrimiento. En términos de Byung-Chul Han (2021), una sociedad algofóbica.

Otra respuesta nos conduce a pensar que el discurso de la medicina ha querido cooptar el dolor sin conseguirlo, porque su presencia es casi incompatible con la positividad de la vida productiva. El dolor no ha sido conjurado, en modo alguno, por la medicina, y menos aún por los fármacos; ninguno puede acabarlo. Resumiendo, algunos elementos importantes en el dolor son: los rasgos biológicos, afectivos y espirituales, su temporalidad, su irreductibilidad ante la muerte, su estado intermezzo -entre la vida y la muerte-, y, finalmente, su resistencia a ser cooptado por otra entidad.

\section{Ética y resistencia, a modo de cierre}

¿Hasta dónde nos ha traído esta lectura foucaultiana? Un primer arribo consiste en poner al dolor en perspectiva dentro de los territorios de la medicina para mostrar que, desde el siglo XVIII, el discurso había girado en torno a la vida, la enfermedad y la muerte. Foucault ratificó la existencia de una economía y un mercado de la salud para estimular la prolongación de la vida a ciertos costos y a favor de la mano de obra productiva, sin crear condiciones que hagan la vida vivible. Este concepto de vida positiva y plena no es compatible con el dolor, ya que en nuestras sociedades contemporáneas “(...) se habla de la mercantilización de la salud, es decir, la reducción del bien humano fundamental de la salud al carácter de mercancía, susceptible de ser sometida al proceso de compra y venta” (Díaz, 2016, p. 109).

Un segundo arribo nos permite observar que posiblemente el discurso de la medicina ha ocultado al dolor porque éste representa una amenaza a la vida positiva y necesaria para la mano de obra. En este sentido, el dolor se distingue de la muerte y de otros males no sólo por su carácter corporal y subjetivo, sino también porque en su vertiente espiritual puede convertirse en un dolor colectivo en el momento en que se instituye como una posible ética 
de la existencia (Sicerone, 2016; Espinel y Pulido, 2017), o una experiencia de la finitud: "El sujeto doliente es capaz de sentir su propia finitud a partir de la presencia del otro (...)" (Cabrera, 2021, p. 397).

En las condiciones imperantes, marcadas por la primera pandemia del siglo XXI, el discurso médico ha instalado el miedo al virus SARS-COV-2 o coronavirus como una amenaza para la vida. Creemos, sin embargo, que el dolor es uno de los últimos reductos disponibles para no sucumbir ante el mercado de la salud ahora convertido en el mercado de las vacunas. Aclaremos que no se trata de rechazar las vacunas anticovid, ni de negar la existencia de la pandemia; se trata de reconocer que el dolor individual y social que ha sobrevenido con la pandemia no puede contrarrestarse con un fármaco; ninguno es capaz de eliminar el dolor biológico ni el dolor espiritual. Es casi imposible ocultar el estado de pérdida y ruptura que el dolor nos ha traído.

Un tercer arribo es relevante para pensar en una ética del dolor que ayude a reconocernos como sujetos dolientes más allá de nuestro propio cuerpo. Hacernos sujetos capaces de sentir el dolor del otro como si fuera en carne propia. Advertimos que no se trata de ponerse en los zapatos de otro, se trata de no sentir que el dolor del otro es ajeno. Ese dolor ya es nuestro: "La ética de Foucault nos invita a la sabiduría, una sabiduría que consiste no sólo en desprendernos de ciertos sistemas de pensamiento y de acción, sino también en romper nuestro silencio ante el sufrimiento que es la triste suerte de nuestra época (...)" (Bernauer, 1999, p. 267).

En las circunstancias actuales nuestro dolor y sufrimiento nos invitan a una ética de la resistencia para no reducir la existencia a la dinámica de la vida productiva. Se trata de resistir ante la presión de la industria del fármaco o el mercado de la salud, y de existir de otro modo honrando la vida vivible y la muerte digna. Insistimos en un ethos que se pone entre los extremos $\mathrm{y}$, en lugar de silenciar u ocultar el dolor, reconocemos que su presencia es un recordatorio de nuestra finitud. Al constituirnos como sujetos dolientes nos damos a la experiencia con otros, y esto significa inducir modos de subjetivación que ponen límite a la medicalización y a la búsqueda de la funcionalidad biológica que nos ha llevado a enfrentarnos con otras formas de existencia.

En este sentido, el dolor no sólo tiene una ética, también tiene una pedagogía que puede inscribir la compasión por el sufrimiento vivido entre nosotros y una ecosofía para reconocer que en nuestro afán por preservar el modo 
de vida productivo hemos causado daño a otros seres (Guattari, 1996). Dicha pedagogía puede concebirse de manera análoga a un dispositivo que permite ver, hablar y reconocer múltiples registros de dolor (más allá de lo biológico, lo médico o anatomofisiológico). Esto implica desarrollar lenguajes y gramáticas que habiliten registros de intelección que desactiven los mecanismos de productividad que han modelado el significado de la vida como fuerza de trabajo o mercancía. Ahora bien, no se trata de pensar únicamente en el dolor humano, pues la ecosofía se presenta como la oportunidad de reconocer nuestra responsabilidad por el sufrimiento planetario. Se trata de redefinir la posición de otras formas de vida distintas de la antropomorfa y crear condiciones más vivibles y dignas para los seres vivienntes.

Finalmente, acudimos a la perspectiva foucaultiana para imaginar herramientas articulables a una pedagogía y a una ecosofía del dolor, con las cuales combatir el mecanismo de explotación de la vida productiva. La crudeza del filósofo francés nos alerta respecto de las dinámicas establecidas en el gobierno de los cuerpos, ya que donde hay poder, hay resistencia.

\section{Referencias}

Bernauer, J. (1999). Más allá de la vida y de la muerte. Foucault y la ética después de Auschwitz. Michel Foucault, filósofo (pp. 254-274). México: Gedisa.

Cabrera, D. (2021). Narrativas neoliberales y modos de subjetivación: sujeto doliente. La educación y el conocimiento bajo el espectro del neoliberalismo en América Latina. Apuntes para entender sus consecuencias sobre las políticas, las instituciones y los sujetos (pp. 375-399). Xalapa: Universidad Veracruzana.

Castro, E. (2016). La verdad del poder y el poder de la verdad en los cursos de Michel Foucault. Tópicos, 31, pp. 42-61. https://doi.org/10.14409/ topicos.v0i31.7959

Castro, E. (2021). Analítica del poder y de la sexualidad. Utopía y Praxis Latinoamericana, 26 (92), pp. 13-29. https://produccioncientificaluz. org/index.php/utopia/article/view/35043 
Clarós, M. y D’Angelo, G. (1994). Diccionario de medicina Mosby. Madrid: Océano.

Díaz, E. (2016). Filosofía de la medicina: la necesidad de una perspectiva crítica en Colombia. Revista Latinoamericana de Bioética, 17 (1), pp. 102-123. https://doi.org/10.18359/rlbi.2018

Espinel, O. y Pulido, O. (2017). Enseñanza de la filosofía. Entre experiencia filosófica y ensayo. Universitas Philosophica, 34 (69), pp. 121-142. https://doi.org/10.11144/Javeriana.uph34-69.efee

Foucault, M. (1984). Arqueología del saber. México: Siglo XXI.

Foucault, M. (1999a). Nacimiento de la medicina social. Estrategias de poder (pp. 363-384). Barcelona: Paidós.

Foucault, M. (1999b). La incorporación del hospital en la tecnología moderna. Estética, ética y hermenéutica (pp. 97-110). Barcelona: Paidós.

Foucault, M. (2005). Las palabras y las cosas. Una arqueología de las ciencias humanas. México: Siglo XXI.

Foucault, M. (2006). El nacimiento de la clínica. Una arqueología de la mirada médica. México: Siglo XXI.

Foucault, M. (2015). La historia de la locura en la época clásica. México: Siglo XXI.

Foucault, M. (2018). La crisis de la medicina o la crisis de la antimedicina. Revista Cubana de Salud Pública, 44 (1), pp. 162-173. https://www. medigraphic.com/pdfs/revcubsalpub/csp-2018/csp181n.pdf

Guattari, F. (1996). Las tres ecologías. Valencia: Pretextos.

Han, B. (2021). La sociedad paliativa. Barcelona: Herder.

Machado, R (1999). Arqueología y epistemología. Michel Foucault, filósofo (pp. 15-30). México: Gedisa.

Mancilla, M. (2021). Ética y estética de la existencia en Michel Foucault. Utopia y Praxis Latinoamericana, 26 (92), pp. 30-43. https:// produccioncientificaluz.org/index.php/utopia/article/view/35044 
Mujica, F. (2020). ¿Pueden los animales sentir dolor? Reflexiones desde la fenomenología. Azafea: Revista de Filosofia, 22 (1), pp. 25-48. https:// doi.org/10.14201/azafea2020222548

Pérez Marc, G. (2011). Cuerpo y subjetividad: una filosofía del dolor. Páginas de Filosofía, 12 (15), pp. 33-54. http://revele.uncoma.edu.ar/ htdoc/revele/index.php/filosofia/article/view/37

Pessoa, F. (1998). El poeta es un fingidor. Antología poética. Madrid: Espasa Calpe.

Pro, M. (2020). Reflexiones sobre el sentido del dolor, el sufrimiento y la muerte, Cuadernos de bioética, 31 (103), pp. 377-386. http://aebioetica. org/revistas/2020/31/103/377.pdf

Raffin, M. (2018). Bio-historia, biopolítica y clínica médica: la producción de lo "humano" en la perspectiva de la medicina moderna según Michel Foucault. Cuadernos filosóficos. Segunda época, 12, pp. 101118. https://doi.org/10.35305/cf2.vi12.39

Ravanal, M. y Aurenque D. (2018). Medicalización, prevención y cuerpos sanos: la actualidad de los aportes de Illich y Foucault. Tópicos, 55, pp. 407-437. https://doi.org/10.21555/top.v0i55.914

Sanguineti, J. (2019). El sentido humano del dolor. Perspectiva filosófica. H. Velázquez y F. Mendoza (Eds.), Antropología del dolor. Un acercamiento interdisciplinario desde la filosofía, biología y medicina (pp. 13-28). Roma: IF Press.

Sicerone, D. (2016). Foucault y la estética de la existencia como praxis libertaria. Debates y controversias. Revista de Filosofia, 33 (82), pp. 23-36. https://redib.org/Record/oai_articulo3198941-foucault-y-laest $\%$ C3\%A9tica-de-la-existencia-como-praxis-libertaria-debates-ycontroversias

Valle, A. (2019). Hacer vivir dejar morir. Procesos de subjetivación entre rendimiento y transparencia. M. Jiménez y A. Valle (Eds.), Sociología y biopolítica (pp. 117-137). México: Universidad Nacional Autónoma de Puebla / Juan Pablos.

Xolocotzi, Á. (2018). La actualidad de la teoría. El papel de la filosofía en una sociedad del rendimiento. Elementos. Revista de ciencia y cultura, 25 (109). pp. 19-25. https://elementos.buap.mx/post.php?id=596 